\title{
SAT.engine: automated planning and validation tools for modern train control systems
}

\author{
B. Wenzel ${ }^{1}$, J. Schuette ${ }^{1} \&$ S. Jurtz ${ }^{2}$ \\ ${ }^{1}$ Dresden University of Technology, \\ Chair of Transportation Systems Engineering, Germany \\ ${ }^{2}$ Ingenieurgesellschaft für Schienenverkehrstechnik mbH, Germany
}

\begin{abstract}
During the worldwide introduction of modern train control system projects, such as the ETCS (European Train Control System), it turned out that the actual engineering processes cannot fulfil the increasing requirements of the new technology. In comparison to conventional train control systems, the amount, as well as the required quality, of planning data is substantially higher. Hence, the effort in all life cycle phases increases significantly. Due to the lack of tools, too many tasks are done in a manual way, which is inherently inefficient and errorprone. Therefore, the Chair of Transportation Systems Engineering at Dresden University of Technology launched the development of the SAT.engine toolbox in cooperation with ISV mbH Berlin eight years ago. SAT.engine stands for "satellite engineering" and provides an efficient method for a satellite based track survey and further processing tools, e.g. for capturing relevant track elements, generating topological plans, producing video simulations for training purposes or the verification and validation tasks of planning data. Besides the SAT.engine, engineering tools and experience, especially the recently developed tools for the automatic validation and verification of trackside data (e.g. ETCS telegrams), will be presented.
\end{abstract}

Keywords: ERTMS, ETCS, verification, validation, planning, tool, satellite, track database, measurement, SAT.engine. 


\section{Introduction}

In order to ensure an acceptable level of risk and high operational quality with complex train control systems, such as the ETCS, an updated and accurate database is essential. Wrongly determined or imprecise distance measures may remain undiscovered for longer times during the processes of planning, realization, approvals and operations which may lead to:

- substantial time and cost efforts in error search

- occasional disturbances during operations

- operational degradation or interruptions

- in worst cases potential hazardous situations

In many projects, a missing database, which is relied upon for planning and engineering, represents a problem.

Another challenge is the vast amount of track data, which has to be planned, implemented and approved correctly. Without the support of tools, there is no way to check the produced track data and complex telegrams in an efficient way. Additionally, missing interfaces and decentralized data management often leads to inconsistencies and loss of data.

Due to the lack of tools and insufficient data quality (cf. figure 1), the actual engineering methods cannot fulfil the increased requirements of complex train control systems like ETCS.

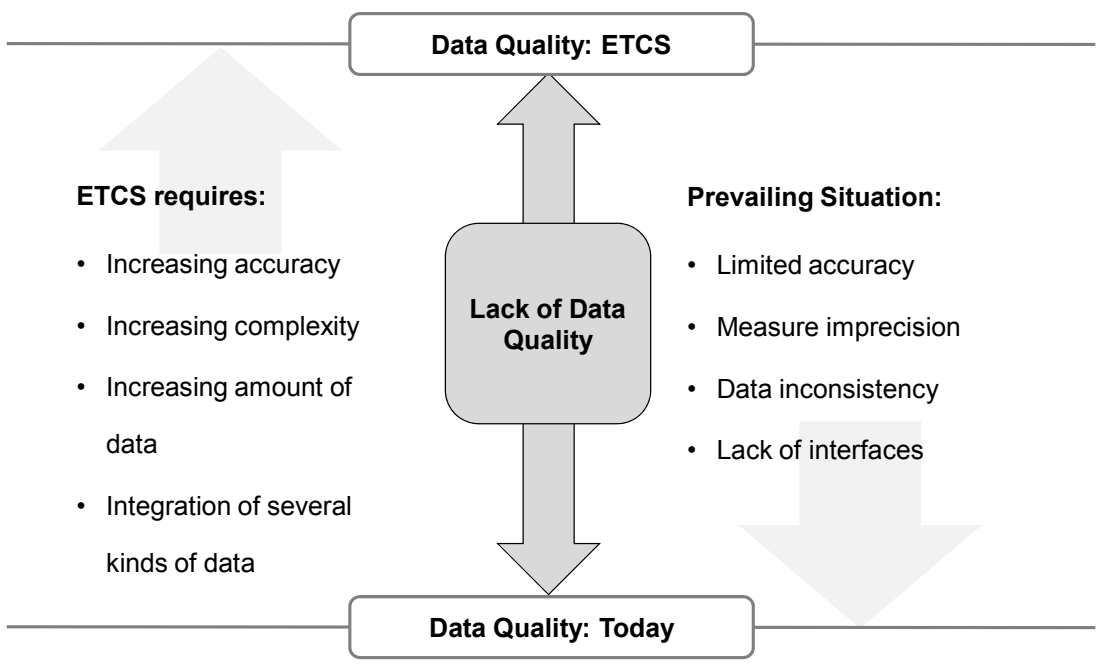

Figure 1: Lack of data quality for ETCS projects. 


\section{Challenges of modern train control systems}

\subsection{Increased requirements for the ETCS}

The ETCS is planned and engineered often into an existing or to be constructed track topology with signalling equipments. The ETCS wayside equipments of all ETCS levels themselves consists of the Individual (Single) Balises (Transponders) or groups of Balises and in some Level 1 cases additionally of Euroloops (Inductive Loops).

Previously, signalling engineering had often been performed on the base of position signs along the kilometre line of the track and in some cases according to distances with respect to particular positions, e.g. danger points. The ETCS, however, needs new, qualitatively higher requirements for the wayside engineering process than could be satisfied by the conventional process.

In the ETCS, the positions and distances refer to the distance that a train actually drives along the track (i.e. with reference to the track centreline) and not the kilometre line. The ETCS onboard units utilize a variety of derived data also for safety relevant functions for which the accuracy of the distances in the plans and in the realization are crucial.

\subsection{Indication of signal equipment positions in signalling plans}

The unambiguous position of ETCS elements is often given by reference to the railway line, its kilometre line and the track. In the case of balise groups, this position relates to a reference point. Track and signalling plans visualize the equipment positions. In such a plan, a balise group is indicated by its identifier and the position with respect to the kilometre reference along the line with a precision of approximately one meter. This value is normally sufficient for identification of the position, but not so for the calculation of the distances along the track centreline.

\subsection{Existing databases}

Errors in the positions and distances of signalling elements are a known phenomenon in existing conventional implementations. A multitude of reasons may account for these deviations, some examples of which may be

- Systematic errors as consequences of non-continuities in the kilometre line ("Milestone-Incongruence"); a position of an element corresponds to the local "kilometre line" without having taken into account a milestone incongruence (also known as mileage change).

- Plans have not been updated after trackworks

- Imprecise of modified measurements during equipment installation.

Figure 2 shows an example of the deviation of a signal position (with reference to the track centreline) and the related value in the signal plan (with reference to the kilometre line). The systematic deviations may be easily recognized. 


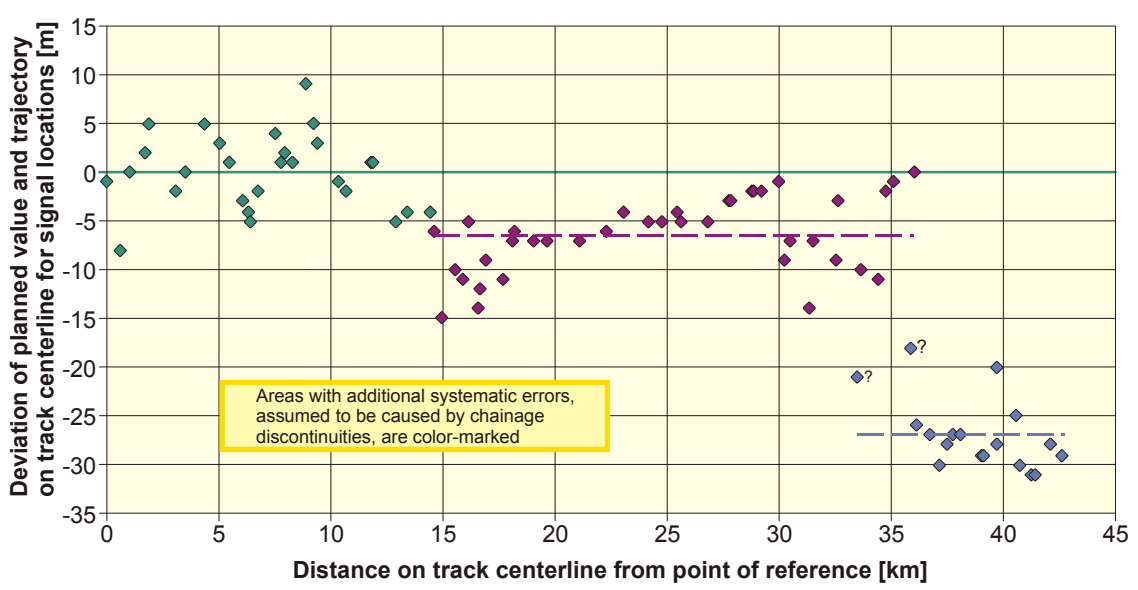

Figure 2: $\quad$ Typical deviations of signal positions.

The visible (random) deviations result from deviating plan values with respect to the kilometre line (objective errors) as well as the differences in lengths originating from the different reference systems (kilometre line next to the track and track centreline in the middle of the track), which represents an additional error in an ETCS project. An ETCS project using these plan values runs significant risks.

\subsection{Determination of distances between signalling elements}

Usually the kilometre line of a two track railway line is situated between the two tracks and, in case of a single track line, in the middle of the track. Nonetheless, there are local deviations from this rule: two track railway lines with numerous curves would yield for an identical position of a balise group different distance lengths in parallel tracks. This effect clearly shows that positions on the base of kilometre lines are not adequate for the calculation of lengths.

In case of existing signalling elements are stored already in a Geographical Information System (GIS) than the required distances may be determined with high precision. The ETCS elements, however, will in general be subject to new engineering and implementation projects and are therefore normally not captured in a GIS system since they do not yet physically exist. The real exact position needs to be determined after installation by a new detailed measurement in order to obtain GIS entries, which translates into additional efforts. Furthermore, the project owners do in many cases not have up to date GIS data available for the existing equipments of a particular section or the entire line.

Also during installation of the balises uncertainties may occur, for example a wrong position of a group of balises due to installation measurement errors. The often used measurement wheel yields position accuracies of about $\pm 1 \%$ when carefully used. The precision will suffer, however, when starting and target point of such measurements have been determined imprecisely themselves, if 
measurement needs to be interrupted or if the rail had not been alternated correctly in curves.

An ETCS project must, however, be based on verified data and installations. For relevant signalling elements in the track, the references to the respective track centreline and amongst themselves need to be known. Furthermore, the distances along the track centreline must be measured correctly. The traditional method for this is a terrestrial precision measurement which provides very precise data that can be stored into a GIS. The basic disadvantage for these full terrestrial measurements and the often required supplementary measurements during realization is the high effort that needs to be invested, in particular for long lines and also possible traffic interruptions.

As an alternative to full terrestrial measurements the SAT.engine tool had been developed.

\section{SAT.engine}

\subsection{The measurement process}

SAT.engine consists of a position acquisition system, a synchronized video stream recording system and an offline SW-engineering environment for further data processing. The system had been jointly developed by the Dresden University of Technology and the ISV $\mathrm{mbH}$.

SAT.engine uses the DGPS (GPS with differential correction) complemented by an Inertial Navigation System. The support positions delivered by DGPS are optimized by the inertial momentum measurement system (accelerations in six directions); also, short discontinuities in the DGPS correction are interpolated by the dynamic measurements without problems. The process yields distance measurements that satisfy the ETCS requirements.

The utilized correction services depend on the geographical situation of the line, the topological conditions and the service availability; typically the system uses services such as OmniSTAR HP/VBS, WAAS/EGNOS or Ascos with correction data transmission over GSM-R or GSM.

In tunnels of up to $450 \mathrm{~m}$ in length, the measurement configuration supplies accurate data; beyond this length additional sensors are required (e.g. odometry).

\subsection{Onboard measurement setup}

Every rail vehicle with a front end cabin (and window) is well suited for a measurement setup. The configuration consists of a few units only: Video cameras are fixed at the screen - for extended purposes also at side windows and loosely cabled; the GPS-antenna needs rapid fixing on top of the vehicle. The compact inertial sensor device requires physical coupling to any solid vehicle part and a laptop computer with the SAT.engine online measurement software coordinates the devices.

Preparation times including calibrations for a measurement does not exceed a couple of hours (e.g. two to four) and dismantling is quick. Measurement travel 
speeds are in the range of $30 \mathrm{~km} / \mathrm{h}$ to $100 \mathrm{~km} / \mathrm{h}$, which provides for effective measurements of also larger distances.

The 315 line kilometres of the ÖBB-Westbahn as an example (Danube Corridor Vienna-Salzburg) had been measured in both directions within two days. Serving for the intended ETCS Level 1 equipment, the positions and distances of and between Pre- and Main Signals had been measured, Marshalling Sign Positions, Switch Tongues, Tunnel Entries as well as platform extremity positions and other data, all with respect to the respective track centreline.

\subsection{Data processing and measurement accuracy}

The measured raw data are further processed by means of particular software tools. The obtained Element Data are visualized and ordered in a Graph Model with vertices and edges. Each edge and every element situated on an edge is identified by an unambiguous data structure; contents and representation of the structures are defined per project. Data are prepared as Element Lists and are stored in a database and/or used with other project engineering tools; also an import/export to CAD tools can be activated.

The recorded video data supplies information on the local surroundings in the course of further engineering and realisation.

Figure 3 shows as an example as snapshot of an Element List. Start and Terminal Nodes of an Edge are constituted by switch tongues (Pxx) of switches (WZ).

\begin{tabular}{|c|c|c|c|c|c|c|c|c|c|}
\hline \multirow{3}{*}{$\begin{array}{c}\text { Station- } \\
\text { Code }\end{array}$} & \multirow{3}{*}{ Nr. } & \multirow{3}{*}{$\begin{array}{l}\text { Track } / \\
\text { line }\end{array}$} & \multirow{3}{*}{ Edge-ID } & \multirow{3}{*}{ Element-ID } & \multirow{3}{*}{$\begin{array}{c}\text { Distance to } \\
\text { start of edge } \\
{[\mathrm{m}]}\end{array}$} & \multicolumn{4}{|c|}{ Delivered for Information only } \\
\hline & & & & & & \multicolumn{2}{|c|}{ GPS Coordinates in WGS 84} & \multicolumn{2}{|c|}{$\begin{array}{l}\text { UTM-Coordinates in WGS } 84 \\
\text { Zone } 39 R\end{array}$} \\
\hline & & & & & & Longitude $\left[{ }^{\circ}\right]$ & Latitude $\left[{ }^{\circ}\right]$ & East value $\mathrm{E}[\mathrm{m}]$ & North value $\mathrm{N}[\mathrm{m}]$ \\
\hline 23 & 1 & L1 & 23-K1_1 & 23-00054IV_1 & $\overline{0,0}$ & $49^{\circ} 32^{\prime} 08.296^{\prime \prime}$ & $25^{\circ} 43^{\prime} 19.445^{\prime \prime}$ & 353096,9 & 2845720,1 \\
\hline 23 & 2 & L1 & 23-K1_1 & 23-00297OR_ & 1048,8 & $49^{\circ} 31^{\prime} 56.706^{\prime \prime}$ & $25^{\circ} 42^{\prime} 47.086^{\prime \prime}$ & 352762,8 & 2844728,1 \\
\hline 23 & 3 & 1 & 23-K1_1 & 23-00055 $\mathrm{HS}^{-1} 1$ & 2046,1 & $49^{\circ} 31^{\prime} 53.217^{\prime \prime}$ & $25^{\circ} 42^{\prime} 14.901^{\prime \prime}$ & 352654,6 & 2843738,9 \\
\hline 23 & 4 & 1 & 23-K1_1 & 23-U1_1 & 2053,0 & $49^{\circ} 31^{\prime} 53.208^{\prime \prime}$ & $25^{\circ} 42^{\prime} 14.677^{\prime \prime}$ & 352654,3 & 2843732,0 \\
\hline 23 & 5 & 1 & $23-K 1$ & 23-WZPO03_1 & 2365,0 & $49^{\circ} 31^{\prime} 52.805^{\prime \prime}$ & $25^{\circ} 42^{\prime} 04.545^{\prime \prime}$ & 352639,6 & 2843420,4 \\
\hline 23 & 6 & 1 & $23-K 2 \_1$ & 23-WZP03_1 & 0,0 & $49^{\circ} 31^{\prime} 52.805^{\prime \prime}$ & $25^{\circ} 42^{\prime} 04.545^{\prime \prime}$ & 352639,6 & 2843420,4 \\
\hline 23 & 7 & 1 & $23-K 2$ & 23-WZP05_1 & 20,6 & $49^{\circ} 31^{\prime} 52.778^{\prime \prime}$ & $25^{\circ} 42^{\prime} 03.875^{\prime \prime}$ & 352638,6 & 2843399,8 \\
\hline 23 & 8 & 1 & 23-K3_1 & 23-WZP05_1 & 0,0 & $49^{\circ} 31^{\prime} 52.778^{\prime \prime}$ & $25^{\circ} 42^{\prime} 03.875^{\prime \prime}$ & 352638,6 & 2843399,8 \\
\hline 23 & 9 & 1 & 23-K3_1 & 23-D3_1 & 96,5 & $49^{\circ} 31^{\prime} 52.656^{\prime \prime}$ & $25^{\circ} 42^{\prime} 00.741^{\prime \prime}$ & 352634,1 & 2843303,4 \\
\hline 23 & 10 & 1 & 23-K3_-1 & 23-00057HS_1 & 103,6 & $49^{\circ} 31^{\prime} 52.647^{\prime \prime}$ & $25^{\circ} 42^{\prime} 00.510^{\prime \prime}$ & 352633,8 & 2843296,3 \\
\hline 23 & 11 & 1 & 23-K3_1 & 23-00059HS_1 & 1432,7 & $49^{\circ} 31^{\prime} 52.520^{\prime \prime}$ & $25^{\circ} 41^{\prime} 17.402^{\prime \prime}$ & 352615,5 & 2841970,0 \\
\hline 23 & 12 & 1 & 23-K3_1 & 23-U3_1 & 1440,1 & $49^{\circ} 31^{\prime} 52.566^{\prime \prime}$ & $25^{\circ} 41^{\prime} 17.166^{\prime \prime}$ & 352616,7 & 2841962,8 \\
\hline 23 & 13 & 1 & $23-K 3$ & 23-WZPOC6_1 & 1539,2 & $49^{\circ} 31^{\prime} 53.180^{\prime \prime}$ & $25^{\circ} 41^{\prime} 13.993^{\prime \prime}$ & 352632,8 & 2841865,0 \\
\hline 23 & 14 & 1 & 23-K4_1 & 23-WZPO6_1 & 0,0 & $49^{\circ} 31^{\prime} 53.180^{\prime \prime}$ & $25^{\circ} 41^{\prime} 13.993^{\prime \prime}$ & 352632,8 & 2841865,0 \\
\hline 23 & 15 & 1 & 23-K4_1 & 23-WZP04_1 & 20,5 & $49^{\circ} 31^{\prime} 53.311^{\prime \prime}$ & $25^{\circ} 41^{\prime} 13.337^{\prime \prime}$ & 352636,2 & 2841844,7 \\
\hline
\end{tabular}

Figure 3: $\quad$ Extract from an element list (four successive edges).

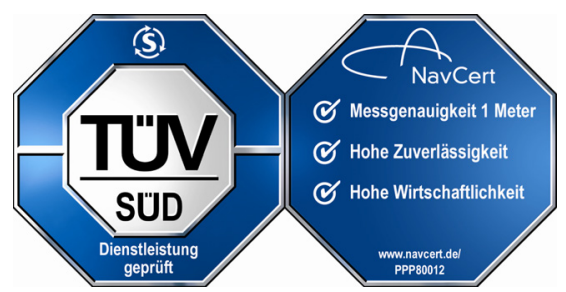

Figure 4: $\quad$ SAT.engine TÜV certificate. 
SAT.engine does not suffer of accumulating errors, all measurement points are situated on the WGS 84 ellipsoid with approximately the same errors. Route length's differences between any elements on any map are provided with comparable uncertainties, independent of the length itself. All distances are supplied with a tolerance of $1 \mathrm{~m}$. This value is based on the experiences of several commercial projects and has been approved by an independent organisation (see TÜV certificate, figure 4). Queries over several plans/maps are possible and will be supported by particular software modules.

The raw position stamped video data is transformed by a special SW tool (SAT.engine.Viewer) to facilitate further planning works. The Viewer software permits the project engineer to easily find and scrutinize particular images of the track at any registered position. The image may then be used for further analysis, e.g. geometrical software modules support the determination of precise position of elements within the viewed image relative to the recorded coordinates of the image itself. The video stream itself may also be "driven" (as play back) by the project engineer in real time or any other speed. In addition, the graph model may be used to invoke images associated with an edge of the model by activating the same in the tool (Figure 5 Example of the ÖBB Westbahn representation in the SAT.engine.Viewer).

\subsection{Constraints and approximations}

In some cases, the accurate positions of some detailed elements may not be properly determined within the video image and require additional terrestrial precision measurements. Two cases had been experienced:

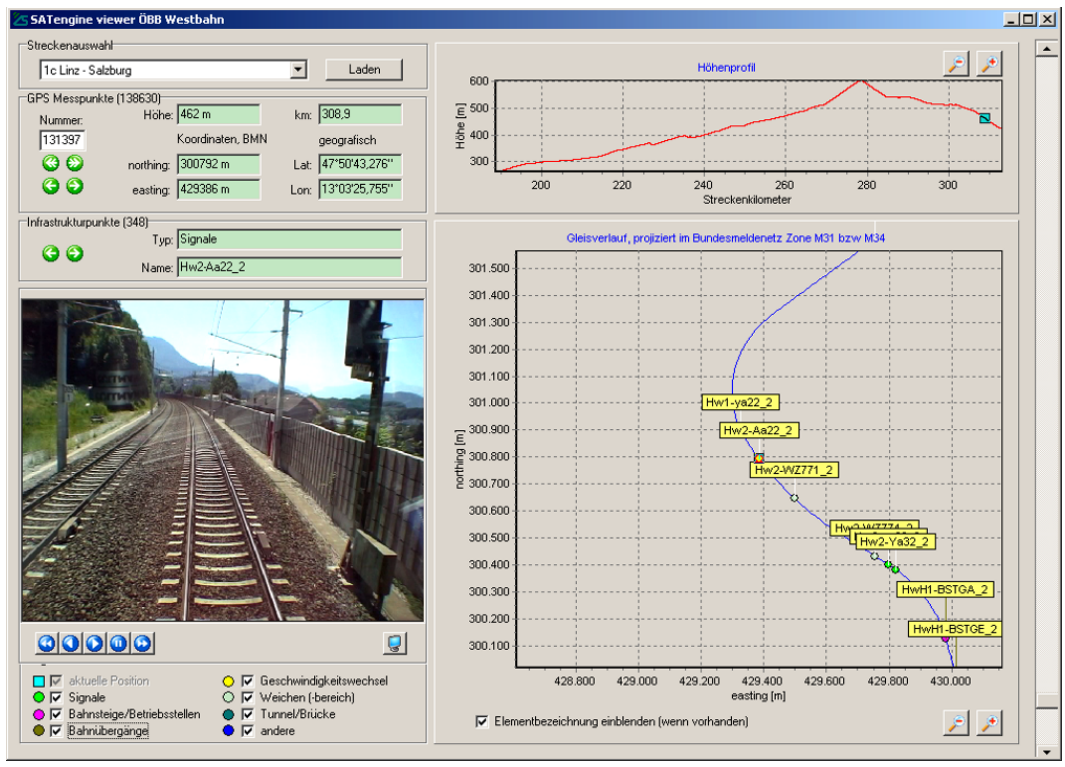

Figure 5: Screenshot of the SAT.engine-viewer. 


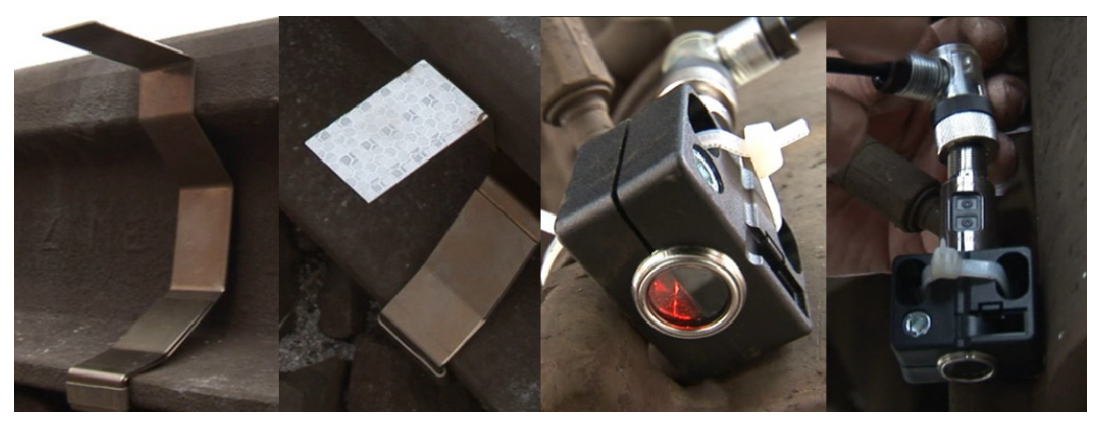

Figure 6: Reflective position marker and detector.

1. Signals in tunnels, at side walls, signal bridges or mounted at mounting extension beams require sometimes additional video photographs in order to accurately determine the positions within/relative to the video image. While recording additional images turns out easy in most events, sometimes an additional terrestrial precision measurement becomes inevitable. The latter can be avoided by previously installed position marks, which are automatically detected during the measurement trip (by reflection, see position marker at figure 6).

2. Measurement in take over or passing tracks requires their accessibility. Should the side tracks not be accessible at measurement times due to civil works or operational reasons, then additional terrestrial precision measurements becomes sometimes more appropriate. The individual measurements are correlated to the SAT.engine measurements at switch tongues that serve as connection vertex.

Track transitions do normally not require additional measurements since synchronization points are normally available in the video stream. Switch tongues as measurement points facilitate the evaluation and latter detailed determination of installation points of the balises with SAT.engine.

\subsection{Use of SAT.engine in the realisation process}

Once the database for an ETCS project had been established and stored, the tool may further also be utilized efficiently during the engineering and implementation phases of the project. Since the application possibilities depend to large extent on the specific project, the subsequent possibilities shall be understood as examples.

- Application during pre-planning and design engineering, e.g. verification of third databases, determination of distances to existing track elements etc.)

- Application during implementation planning and engineering, e.g. determination of any combination of elements, implementation of an ETCS database, etc.) 
- Application during approval, e.g. verification of distances and other attributes of installed elements, validation of distances between balise groups (see 4. SAT.engine Validation).

\subsubsection{SAT.engine in the pre-planning and design engineering}

Here, the recording and processing of ETCS relevant infrastructures with respect to distances between elements is normally of interest. Examples of elements of interest in this context may be:

- Signals locations

- Start of switches or switch tongues respectively, shunting limit signs (if relevant)

- Geographical start and end of platforms

- Tunnel entries/exits

- Relevant danger points if identified and indicated

- Installation positions of route stops.

In general, the to be recorded element list will be prepared by examination of existing track plans or schematic plans, since the early identification of the elements that shall be recorded and have to be expected will facilitate the measurement process. The preparation work leads also to a clear understanding of what must be recorded and evaluated.

In some projects, also the track topology may be recorded for further use in the creation of a signalling track plan itself with SAT.engine (see also 3.5.2).

Verified plans of existing equipments, element lists with the track elements and a track video stream optionally evaluated by SAT.engine.viewer are the results of this phase.

\subsubsection{SAT.engine during implementation planning and engineering}

The limits of the phases "design engineering" and implementation planning/engineering are not commonly and uniquely defined and shall therefore be agreed per country and project.

Based on the previous pre-planning and design engineering, the implementation planning and engineering produces often more detailed plans and models of the ETCS-track elements and their functional cooperation (e.g. balises or balise groups). In general, this means that the element lists will be complemented by the ETCS track elements with increasing levels of detail and information. Experience shows, that during this process it turns out to be beneficial and practical to identify, view, mark and measure the planned location of a new element (e.g. balise) in the track, either completely within SAT.engine or within SAT.engine and in the field. The concurrent and iterative use of SAT.engine for verification and identification of actual (real) locations and the ETCS engineering process yields finally complete and reliable element lists, verified physical feasibility or the later installation and a full graph model (with nodes, edges) of all required distances. 


\section{SAT.engine validation}

Once the installation locations had been identified and measured previously with the tool, the physical installation does not reveal any problems. The question of whether the balises are already programmed or not before installation depends on the signalling company.

After installation of the balises (or, respectively, the Euroloop) a repeated SAT.engine measurement trip for verification represents a very efficient instrument. If the vehicle, used for measurement, is already equipped with the balise antenna (the Balise Transmission Module BTM with an accessible interface), then the SAT.engine verification measurement does not only verify positions and distances to be coherent and consistent with the plans, but may also record the telegram data and check balise identities, orientation and sequence of the balises. The additional features of SAT.engine can be used in particular to facilitate the officially required approval and validation works of the ETCS track equipment.

SAT.engine validation allows the import of several kinds of databases:

- Topology model and element lists of SAT.engine or external measurements

- Recorded telegram data of SAT.engine measurements

- Recorded telegram data of the ETCS Juridical Recording Unit (JRU)

- Route and telegram database of the Radio Block Center (RBC)

- Telegram data files of balises (from Lineside Electronic Units or balise groups)

- Tables/documents of previous planning/engineering phases

During the offline process all available data are imported into the SAT.engine database, which not only stores the collected data but also contains common and project specific engineering rules. In order to support the verification tasks the rules may refer to the imported planning/engineering data. The user selects the rules that apply for the project and all the included telegram data are checked against the defined rules.

Furthermore, it is possible for the user to define individual rules without additional programming effort. Once the checks are finished, the results are stored inside the database as well. The implemented history allows full traceability of all checks done during the approval process and even manages several versions of data or rules. Additionally, SAT.engine validation automatically detects inconsistencies between the topology models of SAT.engine, balise data and RBC database. Thus, for example, linking errors of the installed balise groups with respect to sequence or distances are revealed. The automatic generation of (reduced) track topologic plans on the base of telegram data supports the validation tasks. All tasks may be performed independently of the ETCS Level. 


\section{Further applications of SAT.engine}

\subsection{Gradient profiles}

SAT.engine determines also altitudes of the positions during measurement. The gradient profiles of the tracks may be determined accordingly by evaluating altitude differences between the measurement points. The achieved precision of $\pm 1 \%$ is sufficient for many ETCS projects since anyway only a very small fraction of the measurement values is conservatively utilized.

Figure 7 shows a graphical example of the resulting gradients in a realized project with data in a $25 \mathrm{~m}$ grid. The smaller fluctuations of the measured values may be easily interpolated. As an artefact of the measurement tool, the fluctuation effects are larger at constant gradients compared to changing gradients.

\subsection{Track topology and track topography}

It shall be noted, that in general properly scaled and complete track plans do not always exist when starting an ETCS project, and if they exist, then they may not be useable for the signalling planning or its quality turns out inadequate.

In these cases, SAT.engine allows to generate also a signalling track plan by travelling over the track, evaluating the data and exporting these into a CAD program. In addition, this application had been already successfully performed in an actual ETCS-realization project.

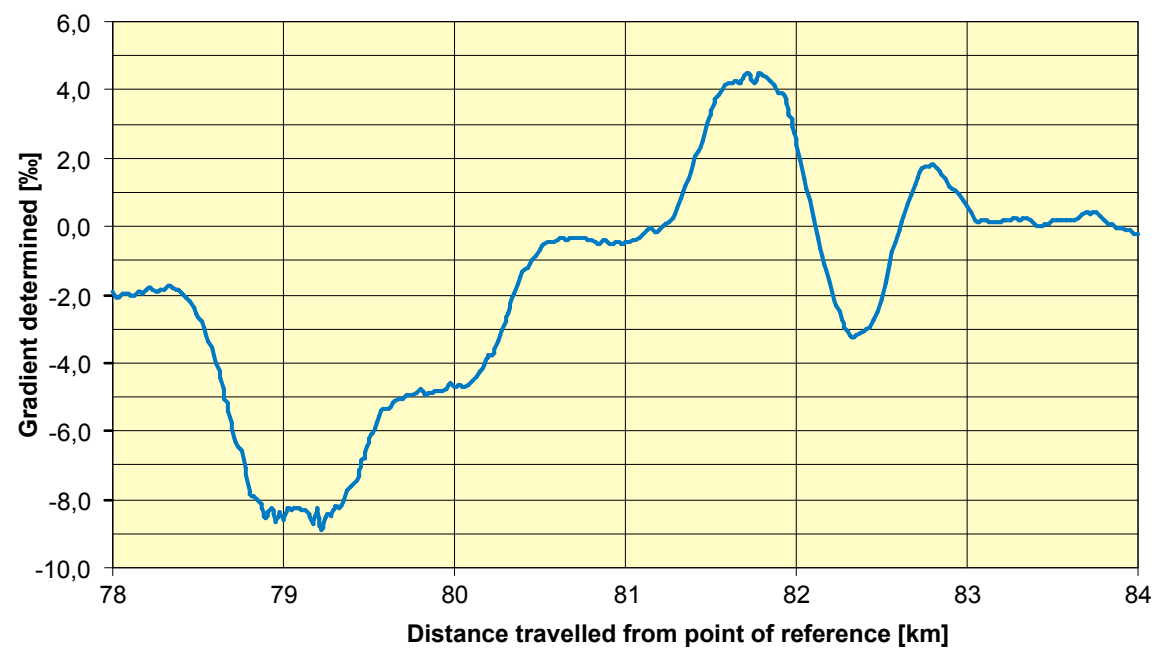

Figure 7: Example for a gradient distribution determined using SAT.engine (extract). 


\subsection{Track simulation with SAT.engine}

In practice, train captain training must often be started while some track sections are still being worked on or still finalized. If at least one track may be recorded previously with SAT.engine then the track video stream can be used as training simulator including new and (virtually) switched signals. Realistic training including variations of speeds and speed profiles, track transitions/routes and signal aspects can be offered by the tool at an early stage.

\subsection{Finding back marked installation locations}

In practice, some installation reference positions of balise groups are indicated by optical marks in the field. Between a measurement/marking and the final installation may, however, pass longer time periods and it has been observed, that some colour marks may not be recognized anymore reliably due to environmental conditions (snow, sand etc.). This turns out particularly problematic if the position is located on a longer track section without any correlation to other local facilities. Since the element lists contain also the geographical positions of the elements, these locations may then be easily be found back locally by GPS personal measurement devices once the process had been accompanied by SAT.engine.

\section{Conclusion}

SAT.engine turns out as a powerful tool for the support of ETCS projects already in its current version. Full performance had been proven in many realization projects. Additionally the automated verification and validation tools seems to be Currently the scope of SAT.engine is extended with the objective of providing a complete seamless and consistent data handing during a complete ETCS project, from the first preparation works until final realization, validation and approval. Furthermore the extension of automated and validation tools will be subject to further research activities. The functionality and practicability of the developed tools will be evaluated during exhaustive tests and realization projects.

\section{References}

[1] Schütte, J., Jurtz, S., Manschewski, H.-W., SAT.engine - an innovative platform to support ETCS-projects. Signal und Draht, 03, pp. 29-36, 2008.

[2] Wenzel, B., Schütte, J., GPS based survey of railway tracks. Proceedings UIC GALILEO Symposium: Paris, 2007.

[3] ERA, Technical specification for interoperability relating to the controlcommand and signalling subsystem of the trans-European high speed rail system and modifying Annex A to Decision 2006/679/EC (2006/860/EC).

[4] ERA, Technical specification for interoperability relating to the controlcommand and signalling subsystem of the trans-European conventional rail system (2006/679/EC). 